\title{
Recognition of alphabetic characters during voluntary eye movements'
}

mILLIAM R. UTTAL AND PAMELA SMITH

THE.UNIVERSITY OF MICHIGAN, ANN ARBOR

In this paper we reconsider the question of vision during eye movements using a novel display procedure which guarantees that the eye was not stopped at any time during the eye movement. The results of our experiment lead us to conclude that true "saccadic suppression," is a most elusive phenomenon. Furthermore, a brief analys is of the optics of the eye movement suggests that a substantial amount the elevation of visual threshold during eye movements can be attributed to simple retinal smear if one acknowledges the dominating importance of edge effects in visual threshold measurements.

A review of the literature concerning visual perception during eye movements quickly reveals an astonishing lack of agreement on what might seem to be the solution to a relatively straightforward question. Briefly stated, the problem is: Does the visual system "see" as well when the eye is moving as when it is standing still? The problem was first formulated at the turn of the century in terms of a controversy which assumed that vision was indeed suppressed during eye movements. The controversy in that context concerned which of two possible mechanisms-retinal blurring or central inhibition-was responsible for the assumed phenomenon. Dodge (1900) championed the notion that the effects were caused by optical blurring, while his antagonist Holt (1903) believed they were due to some more central inhibitory process.

An examination of more recent experiments carried out under a wide variety of experimental conditions by a number of investigators raises a number of issues which go beyond the initial assumptions and formulations of Dodge and Holt. The original assumption that visual perception is blanked or suppressed during eye movements is now challenged by the work of a number of investigators. Krauskopf, Graf, and Gaarder (1966), for example, report no suppression of visual perception during involuntary eye movements. The notion that there is a complete blanking is also questioned by Frances Volkmann (1962) who shows that the effect, though statistically significant, is very small and appears only as a slight increase (.5 log units) in absolute thresholds. Zuber and Stark (1966) and Zuber, Stark, and Lober (1966) show that the suppression which can be measured using either visual thresholds or the pupillary reflex as indicators actually occurs mainly during the period preceding eye movement.
On the other hand, Gross, Vaughn, and Valenstein (1967) report that a complete suppression of the perception of a shift of a bar pattern is reflected in the evoked potential. Latour (1962) reports a complete suppression over a wide range of times both during and preceding the eye movement itself, and Ditchburn (1955) reports complete blanking of a cathode ray trace during involuntary saccades.

These experiments seem to present a picture of a great deal of disagreement but, upon careful inspection of the methodological details and experimental conditions, it becomes clear that a wide variety of different experiments have been subsumed under the general rubric of vision during eye movements. The phenomenon, if it occurs at all, apparently is very much a function of the intensity of the stimulus. A distinction therefore must be made between an elevation of the absolute intensive threshold of the eye to a flash of light per se and a reduction in the ability of the $S$ to process patterned information.

In the current paper we propose to add some new data which, though their specific character cannot resolve the issue finally any better than any of the other experiments, do add further substance to the notion that a generalized blanking during eye movements does not occur and that under some conditions pattern recognition during eye movements is very good indeed. The plan of the experiment which we report here resulted from an incidental observation in our laboratory of the trace on the face of an oscilloscope being used in another experiment. If the sweep amplifier which usually varies the position of the oscilloscope trace along the horizontal axis as a function of time is disconnected, any signal of sufficient amplitude being displayed on the oscilloscope will appear simply as a vertical line. If the eye is scanned horizontally across the face of the oscilloscope, the eye movement itself provides a good substitute for the horizontal scan, and the signal can be seen as spread out as in a normal oscilloscopic display. The perception of this trace therefore depends entirely upon the eye movement.

In the present experiment Ss were asked to recognize alphabetic characters presented in this same way-without a horizontal scan. Rather than the characters being blanked during the eye movement, our results indicate relatively high response levels in this task for brightness levels moderately above threshold. However, the ability of $O s$ to 
recognize alphabetic characters is slightly diminished when the brightness of the stimulus is reduced.

Our results demonstrate a complex pattern-recognition capability during eye movements which argues strongly against the notion of a generalized perceptual blanking. The nature of our stimulus guarantees that there is no obscure cessation of the eye movement $-\mathrm{a}$ criticism which had been made of some of the other studies.

\section{Subjects}

\section{METHOD}

Fifteen undergraduate male Ss with normal vision participated in one phase or another of this experiment. Although all data are presented, our results are mainly adduced for the five Ss who served most regularly in the experiments.

\section{Apparafus}

The $\mathrm{S}$ in each experiment was seated in a darkened, acoustically insulated cubicle. A special cathode ray oscilloscope with an ultrashort persistence P-15 phosphor was positioned $1 \mathrm{ft}$ in front of his eyes measured at the bridge of his nose. The P-15 phosphor has a decay characteristic such that the light emitted after cessation of electron bombardment is reduced to $.1 \%$ of its maximum value in $50 \mu \mathrm{sec}$. Alphabetic characters were plotted on the face of the CRT by means of a point plotting routine controlled by a small digital computer. The set of characters which the $S$ would see under the proper conditions is shown in Fig. 1. Characters were plotted on what would have been a 5 by 7 matrix. However, as we have said, the horizontal coordinates of all points in the matrix were set equal to each other. Therefore all the alphabetic characters plotted appeared to the stationary eye as a single vertical line of dots as shown in Fig. 2. To the unmoving eye the dots themselves varied in apparent brightness depending upon how many points of the 5 by 7 matrix were superimposed on top of each other.

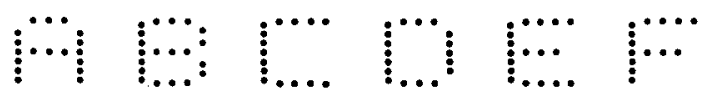

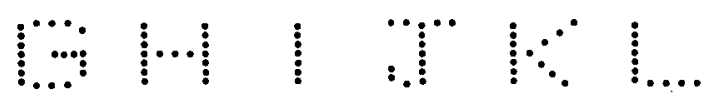

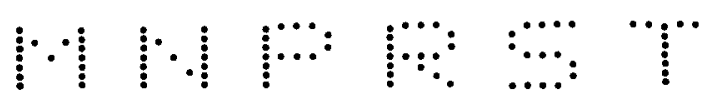

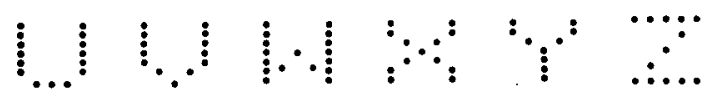

Fig. 1. The character set used in the present experiment. Each alphabetic figure is formed by an appropriate selection of dots from a five by seven matrix.

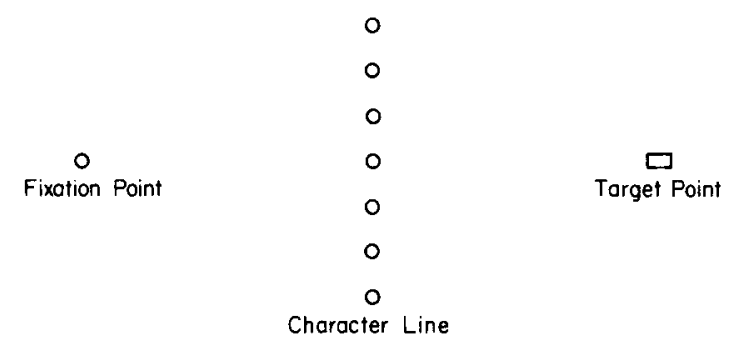

Fig. 2. The display seen by the $S$. The eyes of the $S$ were initially directed at the fixation point. When he moved his eyes towards the target point he triggered the character line. To the unmoving eye, the character line appears as a vertical column of dots, but the dots are spread out into the characters shown in Fig. 1 by the eye movement.

This factor depended upon which one of the 24 alphabetic characters used in this experiment was presented. To the moving eye, however, the single row of dots appeared as a spread-out character since each dot was plotted at a different time.

To synchronize the S's eye movements with the display, electro-oculograms (EOGs) were picked up from two electrodes attached to the temples of the $S$ on a line passing through the pupils of the eyes. This signal was overamplified by two series-connected ac-coupled preamplifiers so that any movement of the S's eyes was signaled as a level shift from a large negative voltage to a ground level voltage at the output of the amplifier. Small eye movements, eye blinks, and possibly other bioelectric potentials, produced spurious level shifts which could occasionally lead to false triggering of the display.

The S's viewing position was standardized by a chin and forehead rest, and the display was observed binocularly throughout the whole experiment. The display observed by the $S$ consisted of three parts as shown in Fig. 2. A fixation point was plotted near the left hand edge of the CRT by the computer. The modified alphabetic display itself appeared near the center of the CRT, and a dim incandescent target light was located near the right-hand edge of the CRT towards which the $\mathrm{S}$ was to move his eyes. The brightness of the first fixation point was adjusted just below the level which resulted in an observable corona around the fixation dot to the dark adapted eye. The brightness of the lines of dots representing the character would be considerably less than the brightness of the fixation dot, since each point in the character would be plotted only once (a $10 \mu \mathrm{sec}$ intensity pulse defined the duration of electron beam bombardment) for each alphabetic character while the fixation point was repeatedly plotted during the pretrial waiting period.

\section{Procedure}

The $\mathrm{S}$ controlled the cocking and triggering of the display mechanism in the following manner. When 
he was ready he would depress a small hand-held push button which would condition the computer to start taking analog to digital conversions of the EOG signal from the templar electrodes. Once cocked, a shift in the EOG from the negative level to the positive level would trigger the release of the display. Barring the involuntary eye blinks and microsaccades mentioned above, this triggering would occur when the $S$ voluntarily moved his eyes from the fixation point on the left to the right-hand target point. The $S$ was instructed to do this with a rapid "crisp" eye movement, and only a few trials were usually necessary to learn the desired technique.

Following the beginning of the eye movement the computer delayed the release of the display by a variable amount of time selected randomly from a table of delays varying from 10 to $160 \mathrm{msec}$ in 10 msec steps.

The selection of each character was also accomplished by means of a random procedure. Only 24 of the 26 characters in the alphabet were used in this experiment- $O$ and $Q$ being considered too much alike in the plotting system we used to be reliably discriminated. Our random procedure was biased so that more of some characters were presented than of others for a nonprocedural reason. The probability of a $U$ was three times greater than any other character, and the probabilities of $E, I$, and $W$ were twice that of the other characters.

In pilot studies the characters were plotted from left to right by columns of the 3 by 5 matrices shown in Fig. 1. If one considers the optics of this situation it is obvious, however, that this leads to a backward plotting of the characters for left to right eye movements. We were then forced to choose between either right to left eye movements or reversal of the order of plotting from left to right to right to left. Because of the cultural bias in our society for left to right reading, it was decided to plot the characters backwards-i.e., each of the five columns of dots was plotted from top to bottom starting at the right. The speed of plotting ( $350 \mu \mathrm{sec}$ between dots) was such that the character appeared as a simultaneously displayed selection of the 35 possible dots in the matrix. The Ss never reported other than simultaneous display as the bits were spread out over the retina by the eye movement. The effect of this backward plotting does, however, show up in the error matrix we shall consider later. The characters displayed were about 1 in. in height at a distance of $1 \mathrm{ft}$ subtending, therefore, a visual angle of approximately $5 \mathrm{deg}$ vertically. Horizontal visual angles depended upon the speed of eye movement, as well as interdot intervals, and therefore varied from trial to trial.

Subjects served for periods of about $50 \mathrm{~min}$ at a time in this experiment. After observing a given display they reported the name of the character observed to the $E$ over an intercommunication sys- tem. The $\mathrm{E}$ typed the S's response into the console keyboard of the computer which automatically responded with a print-out of the delay, the displayed character, and the S's response character. In this way a protocol was logged of the sequence of the experiment. In addition, the computer also punched a paper tape with this same information. These paper tapes were later analyzed by two analysis programs. The first analysis program summarized the data for each $\mathrm{S}$ into two tables. The first table was arranged as a function of the delays between the eye movement and the release of the character. It tabulated the number of times each delay was presented and the percentage correctly identified at each delay. The second table was arranged in alphabetic order. It tabulated the number of times each character was presented, the percentage correctly identified, and also listed the incorrect responses. The second analysis program pooled all the data from all $\mathrm{Ss}$ into two similar summary tables. These latter pooled tables were the basis of most of the analyses discussed below.

At the end of the main experiment described above a supplementary experiment was run to consider the related issue of the effect of stimulus intensity on this form of character recognition. A neutral density filter of $1.4 \mathrm{log}$ units was placed over the vertical column of dots constituting the character, reducing their intensity to a low level. This filter density was chosen since at this brightness level the dots were but barely visible to the stationary eyes. Each of our five primary Ss served for only $2 \mathrm{~h}$ in the supplementary experiments, and data were pooled for all runs using the two analysis programs described above.

\section{RESULTS}

The results of the main experiment are presented in Fig. 3 and Table 1. Figure 3 is a plot of the per-

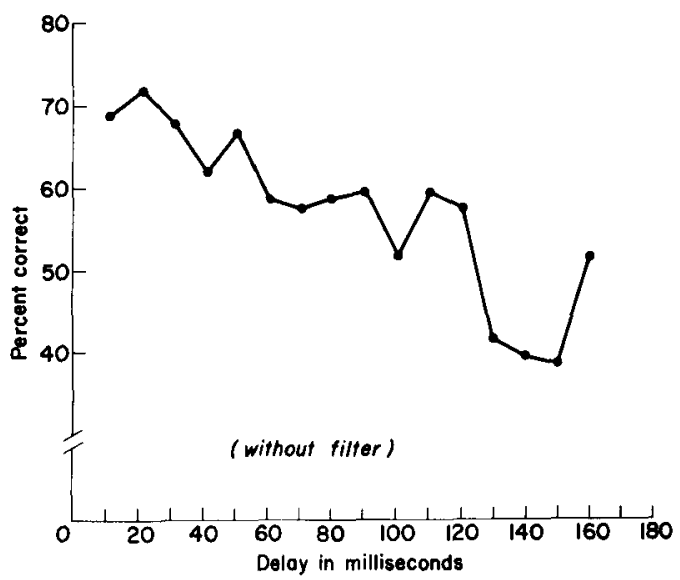

Fig. 3. The results of the main experiment plotted as a function of the interval between the beginning of the eye movement and the release of the character line. The dependent variable is the percentage of the presented characters which were correctly identified. 
Table 1. The results of the main experiment tabulated as a function of the character presented. $N$ indicates the number of times each character was presented. The third column indicates the percentage of each character correctly recognized.

\begin{tabular}{|c|c|c|}
\hline Stimulus Character & $N$ & $\%$ Correct \\
\hline A & 525 & 55 \\
\hline$B$ & 433 & 47 \\
\hline C & 750 & 62 \\
\hline D & 486 & 63 \\
\hline$E$ & 1023 & 68 \\
\hline$F$ & 411 & 62 \\
\hline G & 691 & 32 \\
\hline$H$ & 596 & 54 \\
\hline 1 & 1469 & 89 \\
\hline$J$ & 620 & 60 \\
\hline $\mathrm{K}$ & 561 & 62 \\
\hline$L$ & 353 & 69 \\
\hline$M$ & 522 & 28 \\
\hline$N$ & 466 & 24 \\
\hline 0 & 0 & -- \\
\hline$P$ & 448 & 56 \\
\hline$Q$ & 0 & -- \\
\hline $\mathbf{R}$ & 63.4 & 69 \\
\hline$S$ & 415 & 72 \\
\hline$T$ & 548 & 64 \\
\hline$U$ & 1772 & 57 \\
\hline$V$ & 744 & 35 \\
\hline$W$ & 1153 & 43 \\
\hline$x$ & 493 & 70 \\
\hline$Y$ & 621 & 61 \\
\hline$z$ & 230 & 69 \\
\hline
\end{tabular}

centage of correctly identified characters as a function of the delay between the beginning of the eye movement and the release of the displayed character. Up to a delay of $100 \mathrm{msec}$ there is a gradual decrease in the probability of correct detection of about $10 \%$ from the highest value obtained at $20 \mathrm{msec}$. This effect was not produced by one or a few anomalous Ss; it appears in all of the individual records of the Ss used in the experiment. The performance level is especially depressed in the region from 130 to $150 \mathrm{msec}$.

Table 1 presents the performance information for each of the 24 utilized alphabetic characters and details the number of trials for each character. It can be seen that the characters generally fall into three different groups. The first includes the characters $G, M, N$, and $V$ which were correctly identified in $1 / 3$ or less of the trials. The second group includes the characters $B$ and $W$ which were identified correctly in 47 and $43 \%$ of the trials, respectively. The third group contains all the rest of the characters which were identified correctly in better than half the trials.

Table 2 presents a selected portion of the error matrix for those characters which were in the first two of the three groups. It is important to remember that the error character $I$ is in most cases a triggering error such that the displayed character was released at a time other than during an eye movement. It is the S's way of reporting that he saw a vertical column of dots rather than a spread-out pattern.

Figure 4 presents the results of the supplementary experiments in which the intensity of the display character had been reduced by $1.4 \mathrm{log}$ units. These data clearly show that there is a decrement in performance at this low-light level at all delays although the shape of the function remains the same as in the main experiment. The Ss still, however, report the presence of a spread-out display character indicating that they were not "blanked" during this time. An examination of the related error matrix indicates a relatively small number of I responses. This result suggests that the main cause of the decrement in performance was due to the missing dots. No Ss, even at this low level, reported that they were not able to see anything. All gave some sort of a character as their answer.

\section{DISCUSSION}

We believe that the apparent confusion concerning the question of whether or not vision is possible during eye movements is the result of an inappropriate analysis of the issues involved. Nevertheless, it is quite clear that our visual mechanism does not respond to all of the information actually generated by a stimulus which is blurred by accountable physical factors. A motion picture leads to a relatively smooth and unblurred set of perceptions even though considerable amounts of blur can be seen when one examines one frame in stopped motion. During eye movements additional optical sources

Table 2. A partial error matrix for the characters which were incorrectly identified most often.

An examination of this matrix indicates that the characters most often confused were those with critical features on their right hand side.

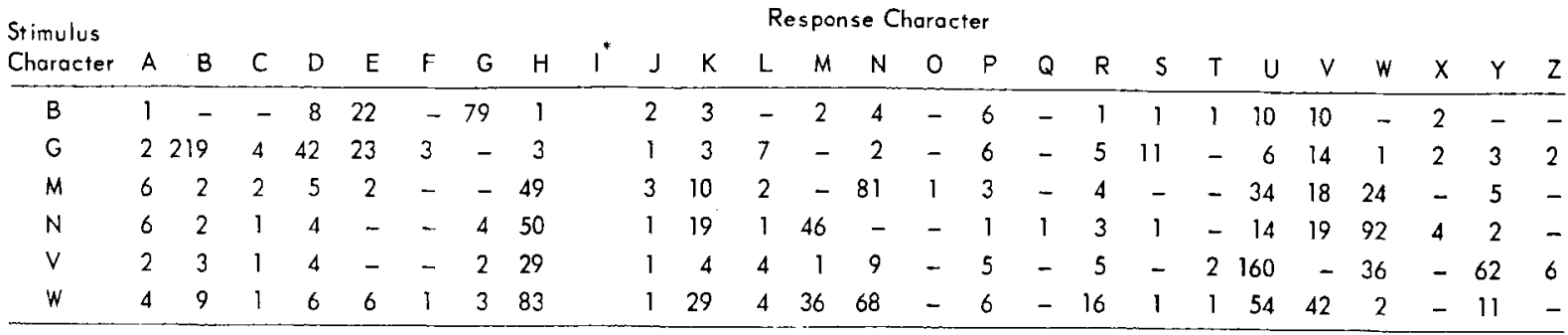

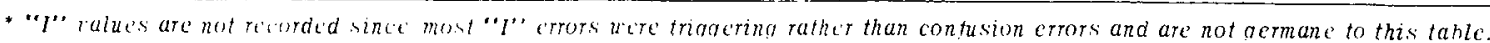




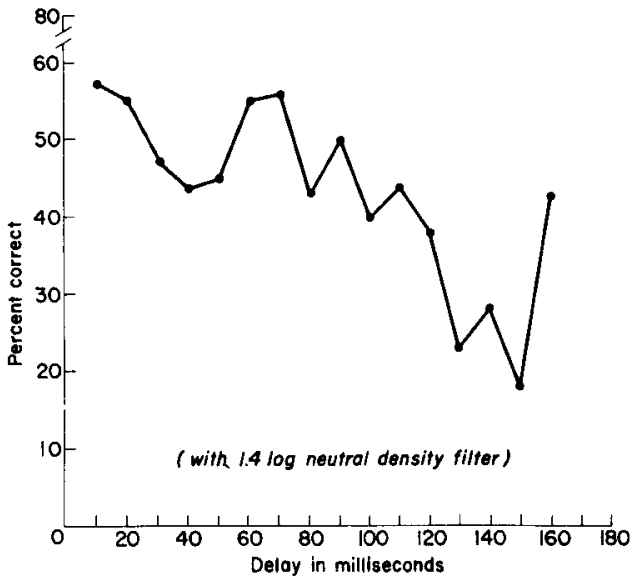

Fig. 4. The results of the supplementary experiment (with a 1.5 log unit filter positioned over the character line) plotted as in Fig. 3.

of blur can be identified, but we do not see that type of blur, either. This has been historically explained by a hypothetical suppression of vision during eye movments. Our survey in the introduction of this paper, however, as well as the results of the present experiment lead us to the conclusion that supposed suppression of vision is a most elusive thing, occurring in the laboratory only under conditions of very low levels of intensity, if at all. Furthermore, if the information content of the signal is reduced to manageable proportions, as in the present experiment, vision during eye movements may be very good. It is important therefore to distinguish in our discussion between a possible suppression of blur at high stimulus intensities characteristic of daylight viewing and the lowering of performance levels at lowered levels of stimulus intensity.

In the main part of this experiment we have been dealing with stimuli which are of the former kind -they are substantially suprathreshold. Our data therefore are not directly comparable to those of experiments in which the concern was with nearthreshold stimuli. The nature of our results suggests that vision is not blanked during eye movements at normal stimulus levels. Our stimulus situation guarantees that our observed results are not due to a momentary stoppage of the eye movement. The suppression of the visual process of high levels of stimulus during eye movements, therefore, is no longer acceptable to us as an explanation of the absence of perceived blur during eye movements. That is to say if we could, in some ideal experiment, implant electrodes in the appropriate locations in the visual pathways we would expect to find that the pattern of neural activity would reflect the physical blurring of the stimulus.

What then can we propose as an alternative to saccadic suppression as an explanation for the lack of awareness of the blurred stimulus? We propose that the explanation is not one based upon a filtering procedure dependent on a reduction in information transmission in the afferent pathways, but rather one based on the limits of information processing at higher centers of the nervous system. This explanatory notion maintains that only a small portion of the tremendous detail of information which is introduced into the nervous system by the sensory processes can be handled by our perceptual mechanisms. In other words, we do not see motionproduced blur, not because vision is suppressed during eye movements, but because blur is extra information which simply exceeds the processing capabilities of our perceptual mechanism. There are many psychological studies which show that even under laboratory situations in which the complexity of the situation has been reduced to a very great abstraction of the real complexity of our environment the limitations on human information processing are very severe. The most famous of these studies is, of course, the work of G. A. Miller (1956). There is no reason to expect that we deal with complex environmental stimuli in a way that allows any very much greater information processing even if multiple dimensions are utilized.

In the light of this limited capacity to process information the perceptual response must be considered as being one which is based upon a minimum amount of new input information, the triggering of prestored patterns, and an insensitivity to missing, redundant, inconsistent, or superabundant information. We feel that there is no basis for hypothesizing blanking in the sense of a reduction in information flow, but rather that the superabundance of information, in a very complex and rapidly changing stimulus environment, is simply beyond the information processing capabilities of the human perceptual system. The human brain treats this extra information in the same way it treats missing information-it ignores it and builds up a hypothesis of the stimulus environment which is consistent with some action of reduced information content. It is an extremely adaptive schema and avoids flooding our perceptual world with a glut of nearly useless and highly redundant information. It is interesting to note that in this sense the limited response rates of most sensory neurons-a maximum of 1000 action potentials per second-as well as the reduction in information content due to anatomical convergence (for example, from the retina to the optic nerve) are useful evolutionary adaptations helping us to remain in symchrony with our environment.

We may also point out the relation of these notions to the rarely discussed problem of how it is that we handle lacunae in our spatial and temporal environments. The absence of stimuli from the blind spot of the retina (even under monocular viewing 
conditions) does not lead to perception of a world with a black hole in it but that lacuna is filled and closed. Similarly, gaps in trains of nerve impulses must be rather large (Uttal \& Smith, 1967) before they become apparent. The point we are making in both of these situations is that the human perceptual processing capability is limited in its ability to respond to the extreme detail available to it through overly efficient receptors. The absence of our awareness of the blind spot gives us an indication of the level of insensitivity with which we live.

Even though we reject the notion of perceptual blanking at high brightness levels, it is necessary to consider the question of the source of the elevation of thresholds when the stimulus intensity is very low. Threshold elevations have been demonstrated by too many experiments in too many different forms to be passed off as simply a set of artifacts. An explanation of this phenomenon can be sought entirely in terms of the varying amount of light falling on the individual receptors during eye movements as compared to when the eye is stationary. The numbers we shall present in the following analysis are only approximations. We have ignored such effects as diffraction and diffusion and other causes which contribute to the blurring of the retinal image even in the unmoving eye. On the other hand there are other factors such as the contrast enhancement due to inhibitory interactions which are known to exist in the visual system and which could, at least in part, compensate for some of those effects. Our analysis may therefore not be too far from a fair approximation.

First, let us make an estimate of the angular velocity of eye movement. Our Ss certainly were still moving their eyes $150 \mathrm{msec}$ after the start of the movement since they were still able to report the character at least occasionally correctly at this delay. However, the dip in the curve between 130 and $150 \mathrm{msec}$ may represent a period of time during which the eye was stopped at a position beyond the target point. The subsequent increase in performance at $160 \mathrm{msec}$ may be due to the movement during the return from this overshoot. Let us, therefore, estimate that the time of motion was about $125 \mathrm{msec}$ to traverse the $25 \mathrm{deg}$ of visual angle between the fixation point and the target point. Using these values, the velocity of eye movement can be calculated to be $1 \mathrm{deg}$ per $5 \mathrm{msec}$ or, since 1 $\mathrm{mm}$ of retinal length is approximately equal to $3 \mathrm{deg}$ of visual angle (Graham, 1965, p. 50), $1 / 3 \mathrm{~mm}$ in $5 \mathrm{msec}$. This is equivalent to $6.6 \mathrm{micra}$ in $100 \mu \mathrm{sec}$. Since the typical diameter of the cross section of a rod or cone is about 1.5 micra, such a velocity suggests that exposure times as short as 10 or $20 \mu \mathrm{sec}$ could allow contours to be shifted across the boundaries of single rods and cones. Thus, the amount of light falling on a given receptor at the contour of a visual pattern could be reduced on the average to about half the available light during this brief period of time. This simple physical analysis is acknowledgedly incomplete, but there is some suggestive evidence from what is known of contour intensification and stabilized retinal images (Ratliff, 1965) that contour effects could dominate in the detection of spot-like visual stimuli. Eye movements may, therefore, be considered as contributing to a reduction in the gradient of the contour, and the effects so produced could be extremely complex in terms of the resultant reciprocal lateral interaction patterns.

Volkmann's (1962) well-designed experiments provide data which probably are the best estimates of the magnitude of the "suppression" effect at low light intensities. Her summary statement concludes that the elevation of the visual threshold in flash detection and character recognition was "about .5 $\log$ units for a $20 \mu \mathrm{sec}$ stimulus flash." Although this is larger than our calculated values-a doubling of the light intensity would be an increase of about $.3 \log$ units-the complexities of the situation and the fact that these two values are not at least of different orders of magnitude suggest that a simple optical explanation-retinal smearing-can account for a considerable amount of the threshold elevation in this type of experiment.

There are still, of course, a very large number of unanswered questions. Our simplistic analysis does not allow us to explain satisfactorily the suppression which occurs prior to the beginning of the eye movement as reported by Zuber and his colleagues. Similarly the changes reported by Gross, Vaughn, and Valenstein (1967) in the evoked potential are curiously disjoint with the data of Volkmann as well as with the reports of Gaarder, Krauskopf, Graf, Kropfl, and Armington (1964) and Bickford and Scott (1967). Both of these two latter studies utilized essentially similar situations to demonstrate phenomena in which the eye movement itself leads to the evocation of the response rather than its diminution. Further, Michael and Stark (1967) report a complex wave shape change associated with suppression rather than the simple reduction in amplitude reported by Gross and his colleagues.

In general the lack of agreement of the evoked brain potential data makes them especially difficult to handle and, even when in agreement, a substantial number of questions have been raised concerning their significance (see, for example, Uttal, 1967).

Another curious discrepant result which we also find hard to explain is Ditchburn's (1955) report that Ss were not able to see signals on the face of the oscilloscope which were triggered by involuntary saccades. Our present experimental design guarantees that the eye is moving during the display 
and recognition of the characters. It is possible that there is some difference between the voluntary and involuntary eye movement. However, Krauskopf, Graf, and Gaarder (1966) also report that vision is not suppressed during involuntary saccades. In his oft quoted report Ditchburn does not discuss the brightness of the oscilloscope trace, and it is possible that his stimulus intensity levels were just too low to be detected by the moving eye on the basis of the retinal smear effects we discussed above. In that case his over-generalization has enjoyed a remarkable persistence.

Let us now consider some of the special details of our current results. The main conclusion that we have come to, of course, is that there is little suppression detectable at moderate light intensities. The reader's attention is now directed to Fig. 3 , in which the performance of our Ss is tabulated as a function of the interval between the beginning of the eye movement and the release of the stimulus characters. The gradual decline in performance over the range of delays is the most noticeable variation. This decline in performance, we believe, is mainly due to differences in the velocity of the eye. The eye movement which the $S$ produces in this experiment is a complex, probably predominantly ballistic, motion in which the first derivative of position-the rate-must go through a maximum at some intermediate position rather than at the beginning of the motion or at the end. The depression of the recognition values for the shortest interval used $(10 \mathrm{msec})$ is probably due to the fact that the eye had not yet accelerated to full speed. Following that early period of acceleration it seems likely that the eye is continuously decelerated in preparation for its arrival at the target point. The effect of the early period of low velocity can also be seen in our error matrix, for it is clear that the characters which were more often mistakenly identified were those characters which had certain critical features on their right-hand side. The righthand side of the character was, because of our reverse plotting technique, the first part of the character displayed. Following this early period, the decline from about $70 \%$ to about $60 \%$ correct identification over the next $120 \mathrm{msec}$ is due, we believe, to the gradual reduction in velocity, since characters would be more spread-out and presumably more easily identified the greater the velocity. The decline is not greater probably because lower velocities, although greatly changing the width of the character, need not necessarily reduce its recognizability until very low velocities are reached.

As mentioned above, the dip in the curve between 130 and $150 \mathrm{msec}$ is also probably due to the dynamics of the motion. An examination of many of the records shown in the various cited experiments shows an overshoot beyond the target point. At the peak of the overshoot the eye movement must be completely halted and thus there would be, on the average, a decreased ability to recognize characters. During the time period the eye moves back to the target point from the overshoot, it would be capable of recognizing some symmetrical displayed characters.

The results of the supplementary experiment with a reduced light intensity are consistent with the conclusions we have drawn above. Although the S's performance level did decrease, there was no breakdown in the general pattern of results. Not only was the original form of the delay function replicated but also Ss never reported an inability to see anything. They continued to give a family of responses indicating that they were seeing well spread-out characters of the same sort observed at the higher stimulus intensities. Rate of error increases probably were due to missing dots rather than pattern suppression.

In conclusion, we find it hard to acknowledge the existence of saccadic suppression. The so-called suppression which occurs at high light levels when the eye moves is more properly described in terms of the limited information processing capability of the perceptual mechanism. During eye movements the amount of variable information being encoded by the receptors greatly increases, but the capability of the perceptual mechanisms to process information remains at its normal level. At low light levels it appears that the small elevation in visual thresholds previously demonstrated by a number of investigators is mainly accounted for in terms of retinal smear.

\section{References}

Bickford, R. G., \& Scott, D. Electophysiologic studies during scanning and passive eye movements in humans. Science, 1967. 155, $101-102$

Ditchbum, R. W. Eye movements in relation to retinal action. Acto Ophthal., 1955, 1 (4), 171-176.

Dodge, R. Visual perception during eye movement. Psychol. Rer., 1900, 7, 454-465.

Gaarder, K., Krauskopf, J., Graf, V., Kropfl, W., \& Amington, J. Averaged brain activity following saccadic eye movement. Science, $1964,146,1481-1483$.

Graham, C. H. (Ed.) Vision and visual perception. New York: John Wiley, 1965.

Gross, E. G., Vaughn, H. G., \& Valenstein, E. Inhibition of visual evoked responses to patterned stimuli during voluntary eyemovements. EEG clin. Neurophy siol., 1967, 22, 204-209.

Holt, E. B. Eye movement and central anaesthesia. Harvard Psychological Studies, 1903, 1, 3-45

Krauskopf, J., Graf, v., \& Gaarder, K. Lack of inhibition during involuntary saccades. Amer. J. Psychol., 1966, 79 (1), 73-81.

Latour, P. L. Visual threshold during eye movements. Vis. Res., $1962,2,261-262$.

Michael, J. A., \& Stark, L. Electrophysiological correlates of saccadic suppression. Exp. Neurol., 1967, 17, 233-246.

Miller, G. A. The magical number seven plus or minus two: Some limits on our capacity for processing information. Psychol. Rev., $1956,63,81-97$. 
Ratliff, F. Mach bands, quantitative studies on neural networks in the retina. San Francisco: Holden-Day, 1965.

Uttal, W. R. Evoked brain potentials: Signs or codes? Perspectives in Biology and Medicine, 1967, 10 (4), 627-639.

Uttal, W. R., \& Smith, P. On the psychophysical discriminability of somatosensory nerve action potential patterns with irregular intervals. Percept. \& Psychophys., 1967, 2, 341-348.

Volkman, F. Vision during voluntary saccadic eye movements. J. Opt. Soc. Amer., 1962, 52, 571-578.

Zuber, B. L., Stark, L., \& Lorber, M. Saccadic suppression of the pupillary light reflex. Exp. Neurol., 1966, 14, 351-370.
Zuber, B. L., \& Stark, L. Saccadic suppression: Elevation of visual threshold associated with saccadic eye movements. Exp. Neurol., 1966, 16, 65-79.

\section{Note}

1. The research reported in this experiment was supported in part by NIH Grant MH08786-3, and in part by NSF Grant GB 6093. We would like to acknowledge the assistance of Mr. Peter Headly and Mr. Richard Nordrum who wrote the data analysis programs. The experimental control program was written by one of us (W.U.) (Accepted for publication December 29, 1967.) 